\title{
Les Francophones et les études postsecondaires
}

\author{
CLAUDETTE TARDIF et FRANK McMAHON*
}

\section{RÉSUMÉ}

Les francophones qui veulent poursuivre des études postsecondaires en français font face à de sérieux problèmes. Cet article examine la condition de l'enseignement postsecondaire offert à la minorité francophone hors Québec en notant d'abord le problème d'accessibilité à des institutions postsecondaires francophones et le phénomène d'assimilation des francophones, pour examiner principalement les effets des institutions postsecondaires mixtes sur les francophones. Les auteurs font une synthèse de certaines recherches et statistiques pertinentes et partagent le fruit de leurs expériences dans l'établissement des écoles francophones homogènes en Alberta.

\section{ABSTRACT}

Francophones intending to pursue post-secondary studies in French face two major problems. The article examines the current status of post-secondary education provided to the francophone minority outside Québec. It first notes the problem of accessibility of French-language post-secondary institutions and the phenomenon of francophone assimilation, and then goes on to study the effects on Francophones of bilingual post-secondary institutions. The authors summarize the relevant research and statistics and share the results of their various experiences in establishing exclusively French-language schools in Alberta.

\section{L'ACCESSIBILITÉ}

A la suite du rapport de la Commission royale d'enquête sur le bilinguisme et le biculturalisme en 1968, il y a eu une reconnaissance du fait que le Canada est un pays officiellement bilingue. La commission était arrivée à la conclusion que tous les Canadiens ont droit à l'enseignement dans leur langue et ce à tous les paliers du système d'éducation. Cependant même 20 ans plus tard, peu d'institutions 
postsecondaires francophones existent à l'extérieur du Québec. Tel qu'indiqué dans le Rapport annuel 1987 du Commissaire aux langues officielles, la majorité des étudiants francophones doivent s'inscrire à des institutions anglophones ou bilingues:

En ce qui les (les francophones hors Québec) concerne, la situation varie grandement selon leur importance numérique dans leur province. Au total, on compte au Canada environ 70 établissements accordant des diplômes universitaires ainsi que 150 établissements d'enseignement collégial; toutefois, en dehors du Québec, il y a très peu d'établissements où l'enseignement est dispensé, ne fut-ce que partiellement, en français. Ainsi les francophones hors du Québec doivent souvent fréquenter une université ou un collège bilingue, ou encore s'inscrire dans un établissement de langue anglaise (p. 220).

Le taux de participation par les francophones aux études postsecondaires a été généralement faible comparativement aux groupes anglophones. Au Canada, les étudiants francophones dans les écoles anglophones ou dans les écoles mixtes ou bilingues ont eu tendance à réussir moins bien sur le plan académique que les étudiants anglophones. Les chiffres du recensement de 1981 indiquent que les francophones n'atteignent pas le même niveau d'éducation que les anglophones. Deux fois plus de francophones que d'anglophones avaient moins d'une neuvième année (30\% comparativement à $15 \%$ ) et ils étaient moins nombreux à avoir complété un niveau académique d'un baccalauréat ou plus $(6,1 \%$ comparativement à 9,1\%). Une étude entreprise par Alberta Advanced Education en 1984 sur les taux de participation aux études postsecondaires révèle que le taux le plus faible de participation (à part les Autochtones) pour les études postsecondaires se situe chez les étudiants (entre 18-24 ans) dont les parents étaient de langue maternelle française.

\section{L'ASSIMILATION LINGUISTIQUE}

Hébert (1989) qualifie de catastrophique la situation de la francophonie dans l'Ouest:

Marginalisée par rapport au milieu social ambiant, décimée par l'assimilation, ravagée par des luttes internes, privée des services sociaux et gouvernementaux les plus fondamentaux dans sa langue, tout semble indiquer son décès imminent ( $\mathrm{p}$. 41).

En effet, les chiffres du recensement de 1981 et 1986 indiquent que le taux d'assimilation dans les provinces de l'Ouest continue à augmenter. En 1986, le taux d'assimilation au Manitoba est de 51,1\% comparativement à 44,26\% en 1981; en Saskatchewan le taux est de $72,5 \%$ en 1986 comparativement à $63,75 \%$ en 1981 et en Alberta le taux est de 67,4\% en 1986 comparativement à $57,6 \%$ en 1981.

Très peu d'étudiants francophones dans l'Ouest sont des finissants des écoles francophones, c'est-à-dire qu'il existe très peu d'écoles ou les francophones 
peuvent recevoir totalement un enseignement dans leur langue dans un milieu qui renforce leur identité socio-culturelle. L'Alberta, par exemple, ne compte que quatre écoles entièrement françaises en 1989-90. Pour la première fois, en septembre 1989, un programme complet au niveau secondaire 10-12 voit le jour à l'école Maurice Lavallée. Il y a donc très peu de francophones qui entrent au niveau postsecondaire avec une formation académique adéquate en français.

\section{LES EFFETS DES INSTITUTIONS MIXTES}

La popularité des programmes d'immersion à travers le Canada a augmenté le nombre d'élèves désireux de poursuivre des études postsecondaires en français. A la Faculté Saint-Jean de l'Université de l'Alberta, les statistiques des inscriptions des deux dernières années indiquent que la proportion d'étudiants anglophones comparativement aux étudiants francophones augmente et, pour la première fois, a même dépassé le nombre d'étudiants francophones. (voir tableau).

\section{Tableau}

STATISTIQUES LANGUE MATERNELLE

FACULTÉ SAINT-JEAN

\begin{tabular}{|c|c|c|c|c|c|}
\hline & \multicolumn{2}{|c|}{ Nouveaux étudiants } & & \multicolumn{2}{|c|}{ Inscription totale } \\
\hline & $1987-88$ & $1988-89$ & & $1987-88$ & $1988-89$ \\
\hline Français & 72 & 63 & Français & 243 & 234 \\
\hline Anglais & 117 & 115 & Anglais & 228 & 264 \\
\hline Autres & 4 & 8 & Autres & 24 & 32 \\
\hline Inconnue & 0 & 2 & Inconnue & 10 & 6 \\
\hline Total & 193 & 188 & Total & 505 & 536 \\
\hline
\end{tabular}

Puisqu'un grand nombre d'étudiants francophones en situation minoritaire doivent fréquenter des institutions mixtes au niveau postsecondaire, il nous a semblé opportun d'examiner la validité de cette formule pour répondre aux préoccupations d'accessibilité et d'assimilation.

Quels sont les effets pour les étudiants francophones d'une institution mixte? ${ }^{1}$ Beauchemin (1985, p. 63) considère que "l'impact des élèves d'immersion dans une institution bilingue doit être abordé à deux niveaux, à savoir: l'impact quantitatif et l'impact qualitatif de l'intégration des 'immergés' aux cours et programmes en français."

L'aspect quantitatif risque fort de favoriser une formule d'institutions mixtes: nombre suffisant d'élèves pour un éventail de cours plus large et un appui financier plus fort. En est-il de même pour l'aspect qualitatif? Les études de cette formule mixte au niveau postsecondaire sont peu nombreuses. Par contre la question de l'école française a suscité une importante littérature historique et plus récemment plusieurs études scientifiques. Deux études importantes, celle du Manitoba (Hébert, 1976) et celle pour l'Ontario (Churchill et al., 1985) justifient de grandes inquiétudes pour les jeunes francophones inscrits dans les écoles mixtes 
(programme anglophone et francophone). Les études de Landry et Allard (1984, 1987a, 1987b) comme celle de Cummins (1986) nous indiquent que la situation minoritaire au plan sociologique exige que le jeune francophone puisse avoir accès à une éducation dans une institution scolaire aussi homogène que possible (i.e. orientée exclusivement vers le service de jeunes francophones) afin de se voir assurer une égalité de chances à l'école.

Alors que les études nombreuses sur l'immersion indiquent que le jeune anglophone majoritaire peut accéder à la maîtrise d'une deuxième langue qui vient s'ajouter à sa maîtrise croissante de l'anglais (bilinguisme additif), le jeune francophone semble perdre son français à mesure qu'il est exposé à l'anglais (bilinguisme soustractif) à moins de se trouver dans une situation scolaire très française. Il a même été proposé que ce bilinguisme soustractif peut conduire à une forme de semi-linguisme, phénomène qui expliquerait la réussite scolaire relativement faible des francophones.

Ces études dépendent surtout de situations où des programmes anglais accueillent des élèves francophones ou bien coexistent avec des programmes français. La seule étude que nous connaissions qui aborde directement la situation où des élèves d'immersion sont mêlés avec des élèves francophones indique, toutefois, la même tendance (Carey, 1987).

L'article 23 de la Charte, qui devait régler le problème de l'école française à l'extérieur du Québec n'a pas envisagé la situation des élèves d'immersion. Par contre, à peu près tous les francophones en dehors de la ceinture bilingue devront se décider devant des écoles mixtes, étant donné l'intérêt pour l'immersion, et la dispersion géographique des francophones qui permet difficilement des établissements distincts.

En ce qui concerne le secteur postsecondaire qui a évidemment une autre dynamique et un autre contexte que les écoles publiques, nous voyons deux genres de question comme étant importants dans la problématique d'une institution mixte au niveau postsecondaire: 1) la qualité de la langue et 2) les valeurs et l'idéologie culturelles. C'est devant ces questions que nous avons examiné l'expérience des parents d'Edmonton.

A la suite de l'adoption de l'article 23 (1982), un groupe de parents à Edmonton obtenait l'établissement d'une école élémentaire francophone (1984). Puisque cette école devait desservir une population de langue maternelle française de 24710 personnes dans la région métropolitaine d'Edmonton, et qu'une étude démographique (Zdunich, 1988) indiquait 2965 étudiants de langue maternelle française en âge de fréquenter une école secondaire, la nécessité d'une école secondaire francophone semblait s'imposer. La difficulté principale pour obtenir cette institution, malgré l'abondance de locaux disponibles pour la Commission scolaire (les écoles du centre-ville s'étant vidées avec le vieillissement de la population), était l'existence d'une école secondaire mixte dans laquelle deux tiers des élèves étaient francophones et un tiers étaient d'immersion. Cette école avait été fondée en 1971 pour desservir les élèves francophones selon le régime de l'époque, où on permettait l'enseignement en français pendant $50 \%$ de la journée. 
Ce qui nous apparait important dans nos considérations pour le postsecondaire, c'est que la Commission scolaire décida d'abord de déclarer cette école mixte une école francophone selon la Charte mais devant accueillir en même temps les élèves d'immersion. A la suite de cette décision, les parents francophones mirent sur pied un plan d'action pour franciser l'école mais qui fut refusé par les autorités de la commission scolaire.

Les recommandations suivantes furent l'objet du refus. Premièrement, les parents francophones tenaient à ce que le comité consultatif des parents fonctionne en français. Ils voyaient mal comment des parents d'immersion étaient compétents pour déterminer l'aspect communautaire de l'école si celle-ci devait répondre aux exigences de la Charte. Deuxièmement, les parents francophones tenaient à regrouper les élèves à l'intérieur des classes en fonction de leur appartenance ethno-culturelle plutôt qu'en fonction d'une compétence linguistique établie de façon administrative. Troisièmement, les parents francophones tenaient à ce que les autorités scolaires continuent à chercher un autre site où on pourrait éventuellement localiser le programme d'immersion.

Le refus des autorités de la commission scolaire d'accepter ces recommandations représentait la pression politique exercée par les parents des élèves d'immersion. Nous citons le texte d'un des surintendants ${ }^{2}$ qui répondait à la demande des parents francophones: “... by publicly stating that we will continue to pressure the anglophone students to relocate to another school would not signify good intentions as interpreted by immersion parents in my opinion."

L'idéologie du "parent d'immersion" se manifeste lorsque le "Bilingual Advisory Committee" (comité de parents d'immersion) indiquait dans son mémoire à la commission scolaire que les parents anglophones souhaitaient pour leurs enfants d'être exposés à la mosaïque multiculturelle et multiethnique. Cette idéologie se voit encore par cette lettre ${ }^{3}$ d'un président de comité de parents d'immersion qui dit: "if the Francophone culture is threatened by maintaining friendly contact with immersion students ... there is a real question of whether or not it is a culture that can be preserved ..."

La pression politique exercée par les parents des élèves d'immersion dans la question de l'école secondaire francophone à Edmonton démontre bien la vulnérabilité des francophones en situation minoritaire. Le refus de la commission scolaire d'établir un programme francophone secondaire dans des locaux convenables pour une population possible de 3000 élèves et d'accorder par la suite une école bâtie pour plus de 1000 élèves à une clientèle de 200 étudiants d'immersion suggère une idéologie qui, malgré sa sympathie réelle pour la langue française, n'a rien compris du vécu des Franco-albertains, cette façon d'être et de sentir qu'on appelle généralement sa culture.

\section{QUALITÉ DE LA LANGUE}

Une question qui se pose lorsqu'il s'agit de l'intégration des “immergés" avec les francophones est celle de la compétence linguistique des deux groupes. Plusieurs 
recherches (Richmond, 1984; Harley, 1984; Swain et Lapkin, 1984) confirment les limites de l'immersion. Les étudiants issus des écoles d'immersion, quoiqu'étant bien supérieurs dans leurs compétences langagières aux étudiants de programmes traditionnels de français, ne possèdent pas une compétence équivalente à des locuteurs natifs francophones. Il y a beaucoup de différences dans la spontanéité, dans la variété et la quantité de leur vocabulaire et généralement dans tout le domaine de la production orale et écrite.

Le genre d'erreurs faites par les élèves d'immersion comparativement aux francophones diffère. Selon Harley (ibid, p. 61), les premiers ont des compétences sociolinguistiques et grammaticales inférieures à celles des élèves francophones. La compétence linguistique de trois groupes d'élèves franco-ontariens, a fait l'objet de plusieurs études par Mougeon, Heller, Beniak et Canale (1984). Ils affirment :

... on a pu constater qu'à niveau de scolarité égal, les élèves d'immersion commettaient plus d'erreurs que les (francophones) anglo-dominants et à plus forte raison que les franco-dominants et les bilingues (p. 237).

Ils ajoutent:

... les enseignants sont d'avis qu'ils (les anglo-dominants) ont un effet retardant sur les franco-dominants pour ce qui est de l'apprentissage du français et des autres matières. (p. 346)

Cet "effet retardant" sera donc plus prononcé par la présence d'élèves d'immersion.

Une des grandes faiblesses et déceptions des programmes d'immersion est le manque de motivation qu'ont les élèves sortant de ces programmes à nouer des liens avec les francophones. D'après certaines enquêtes (Burns et Olson, 1981; Morrison et Bonyun, 1985; Genesee, 1988), il ressort que les élèves de l'immersion ne manifestent pas un plus grand désir de rechercher des occasions pour employer le français dans leur communauté. La vision d'un Canada uni par un bilinguisme national semble remplacée par des considérations beaucoup plus pragmatiques telles que des meilleures perspectives d'emploi, la supériorité des programmes, etc. Selon Bibeau (1988), l'enseignement immersif considère l'apprentissage de la langue comme indépendant de l'expérience culturelle. Dans le même sens, Calvé (1986) considère l'absence de culture dans les programmes d'immersion comme le talon d'Achille de tout l'enseignement immersif. Calvé (1986, p. 27) affirme qu' "il y a vraiment danger qu'on produise, en immersion, des générations d'étudiants qui n'ont jamais saisi la spécificité culturelle, conceptuelle, du français, qui n'ont jamais saisi comment les francophones voient, pensent et découpent le monde et surtout, qui n'ont jamais appris à vivre au contact des francophones." 


\section{VALEURS ET IDÉOLOGIE CULTURELLES}

Les recherches en immersion et l'expérience des parents francophones d'Edmonton soulèvent donc toute la question de l'épanouissement culturel et linguistique des francophones dans un milieu mixte où il y a des finissants des programmes d'immersion. Les élèves d'immersion n'auraient pas le même sentiment d'appartenance à l'histoire, au système de valeurs, aux coutumes, aux institutions et à la langue qu'ont les élèves francophones. L'élève d'immersion s'intéresse à la langue française sans incorporer les éléments culturels de cette communauté, qui sont tous conditionnés par le vécu minoritaire.

Le francophone se voit comme faisant partie d'une communauté nationale d'expression française ayant énormément de mal à survivre et à s'épanouir. Le maintien de son groupe ethnolinguistique est fortement menacé. Il est assiégé par la langue et la culture anglophones. En conséquence, l'individu a tendance à s'associer à la langue et à la culture qu'il perçoit comme étant plus prestigieuses. Le bilinguisme qui en ressort est souvent de nature soustractive (Lambert, 1977; Cummins, 1986; Landry et Allard, 1984, 1987), c'est-à-dire que la deuxième langue déplace sa langue maternelle et lui nuit. L'élève d'immersion, par contre, appartient au groupe majoritaire: il se voit accédant à des statuts socioéconomiques supérieurs de par sa langue maternelle et son accès aux avantages que lui procure son bilinguisme.

Il est intéressant de noter ici que le chapitre albertain de Canadian Parents for French s'est entendu avec l'Association Canadienne Française de l'Alberta (1986) pour défendre la nécessité d'assurer des institutions distinctes pour les deux clientèles. Alors que l'école française se dote d'enseignants activement impliqués dans la communauté francophone, on ne demande pas ce genre d'engagement chez le personnel d'immersion. L'école française se doit de chercher "à renforcer le sentiment d'identité culturelle et linguistique de l'élève francophone ainsi que son appartenance à la communauté francophone de l'Alberta, du Canada et du monde"; l'école d'immersion cherche à "respecter l'identité et l'intégrité culturelles et linguistiques de l'élève."

L'apprentissage de la langue maternelle et d'une langue seconde ne peut être dissocié du processus d'identification de l'individu, ainsi que des valeurs qu'il place sur les deux langues. Selon Landry et Allard (1984), le minoritaire possède un système de croyances vis-à-vis de la vitalité de sa communauté et ces croyances sont à la base du comportement langagier. Si le minoritaire attribue une valeur négative à l'appartenance à ce groupe, cela peut devenir une source de conflits d'identité. Il peut se produire chez le minoritaire un sentiment d'infériorité, et un déchirement psychique car il ne sait plus à quel groupe s'identifier. Selon Fitouri (1983):

Qu'un bilingue fasse des associations d'infériorité ou de honte avec l'une des langues qu'il parle, cela peut avoir pour lui, quand il s'exprime dans cette langue, 
des effets préjudiciables ... Il se peut qu'il se sente, par exemple, ridicule aux yeux des autres quand il emprunte cette langue et cela peut constituer pour lui une interférence intellectuelle dans cette langue (p. 100).

Les taux élevés d'assimilation démontrent bien les choix qui sont souvent faits par les individus qui ne possèdent pas un réseau de contacts linguistiques bien développé dans leur langue maternelle et qui attribuent une valeur négative à l'appartenance au groupe francophone.

\section{CONCLUSION}

Evidemment, l'institution postsecondaire fonctionne de façon différente que les écoles primaires et secondaires. Les étudiants qui y accèdent sont normalement d'âge adulte. Les autorités institutionnelles ont une marge d'autonomie dans la prise de décision des programmes, des conditions d'admission, des mécanismes d'évaluation, etc. Toutefois, quand ces institutions sont francophones, elles ont un rôle important à jouer auprès de la communauté. Privés de la plupart des institutions d'une collectivité nationale, il est normal que les francophones hors Québec s'identifient avec leurs centres francophones de façon tout à fait privilégiée. Dans un sondage auprès de la population francophone d'Edmonton, la Faculté Saint-Jean était identifiée comme l'appui principal de la communauté francophone par la majorité des répondants (McMahon, sous presse).

Cet appui de la francophonie peut disparaître si l'institution se redéfinit. Les jeunes francophones qui y accèdent, provenant pour la plupart d'écoles mixtes ou anglaises, ont l'habitude de rendre leur francophonie anonyme. Les jeunes d'immersion, par contre, se croient parfaitement en mesure de dicter à qui veut les entendre ce qu'est la culture française au Canada, étant des produits de nos institutions bilingues. Il est loin d'être sûr que les institutions francophones puissent maintenir leur identité culturelle devant un nombre important d'étudiants d'immersion.

Malgré les difficultés très réelles que cela peut représenter, la logique de nos réflexions exige une remise en question très sérieuse d'institutions postsecondaires mixtes au sens d'étudiants francophones et anglophones (même d'immersion). Il y a un risque sérieux de gagner la bataille mais d'y perdre la guerre. L'expérience albertaine démontre que les valeurs et l'idéologie culturelles des deux groupes ne sont probablement pas compatibles. Les recherches indiquent que l'enseignement en français ne suffit pas pour renforcer l'identité culturelle du francophone en milieu minoritaire. Il faut créer un milieu spécial qui viendra contrebalancer les effets du milieu dominant anglophone et qui répondra aux besoins linguistiques, culturels et éducatifs des francophones.

\section{NOTES}

1 Le Blanc (1988) définit l'école mixte, comme l'institution où la clientèle est composée d'étudiants francophones et anglophones qui reçoivent l'instruction dans leur langue respective mais qui 
partagent les mêmes locaux et la même administration. Les écoles d'immersion qui regroupent dans les mêmes locaux des étudiants francophones peuvent être considérées des écoles mixtes puisqu'elles répondent aux besoins linguistiques, culturels et éducatifs de deux clientèles distinctes.

2 Lettre reçue par la Société des parents pour les écoles françaises d'Edmonton (avril 1986).

3 Lettre reçue par la Société des parents pour les écoles françaises d'Edmonton (avril 1988).

\section{REFERENCES}

Alberta Advanced Education. 1984. Participation Patterns Studv. Report of the Committee to Examine Participation Trends of Alberta Post Secondary Students. Edmonton: Alberta Advanced Education Planning Secretariat.

Association canadienne-française de l'Alberta et Canadian Parents for French Alberta Branch. 1986. Education française: Deux clientèles, deux programmes.

Beauchemin, C. 1985. "L'impact négatif de l'arrivée des élèves d'immersion. Actes du Colloque national sur l'enseignement postsecondaire en langue française à l'extérieur du Québec. Fédération des Francophones hors Québec, Ottawa. 63-67.

Bibeau, G. 1988. "L’immersion, une formule pédagogique à repréciser." Québec Frangais, 70: 32-33.

Bilingual Advisory Committee, Edmonton Catholic School Board. 1987. Mémoire présenté au Edmonton Catholic School Board, décembre.

Burns, G., Olson, P. 1981. Implementation and Politics in French Immersion. Toronto: Ontario Institute for Studies in Education.

Calvé, P. 1986. "L'immersion au secondaire: bilan et perspective." Contact, 5 (3), 21-28.

Canada, Statistique Canada, Recensement du Canada de 1981. Ottawa, Ministère des Approvisionnements et Services, 1983.

Canada, Statistique Canada, Recensement du Canada de 1986. Ottawa, Ministère des Approvisionnements et Services, 1987.

Carey, S. 1987. "Reading Comprehension in First and Second Languages of Immersion and Francophone Students". Canadian Journal for Exceptional Children, 3 (4): 103-108.

Churchill, S., Frenette, N., \& Quazi, S. 1985. Education et besoins des Franco-ontariens: Le diagnostic d'un système d'éducation. Toronto: Le conseil de l'éducation franco-ontarienne. volume 1 et 2 .

Commissaire aux langues officielles, Rapport Amnuel 1987: Le Renow'eau, égalité. continuité. Ottawa. Ministère des Approvisionnements et Services, 1988.

Commission royale d'enquête sur le bilinguisme et le biculturalisme. I'Education (vol, 2). Ottawa. Imprimeur de la Reine, 1968.

Cummins, J. 1986. "Empowering minority students: A framework for intervention". Harvard Educational Review, 56: 18-36.

Fédération des jeunes Canadiens français, Fédération des francophones hors Québec et la Conmission nationale des parents francophones. 1988. Vers l'égalité des chances. L'éducation postsecondaire en français hors Québec, positions respectives présentées au Secrétariat d'Etat du Canada.

Fitouri, C. 1983. Biculturalisme, Bilinguisme et Education. Paris: Delachaux et Niestle.

Genessee, F. 1988. "L'immersion française: une histoire à succès." Québec Français, mai, 70: 28-30.

Harley, B. 1984. "Mais apprennent-ils vraiment le français?" Langue et Société, 12: 57-63.

Hébert, R. et al. 1976. Rendement académique et langue d'enseignement chez les élèves francomanitobains. Manitoba: Centre de recherche du Collège Universitaire Saint Boniface.

Hébert, R. 1989. "La francophonie dans l'Ouest: Déboires et victoires." Langue et Société. Dossier spécial, été 1989.

Lambert, W. 1977. "The Effects of Bilingualism on the Individual: Cognitive and Sociocultural Consequences." Dans P.A. Hornby (Ed.), Bilingualism: Psychological, Social and Educational Implications. New York: Academic Press. 
Landry, R., Allard, R. 1984. "Bilinguisme additif, bilinguisme soustractif et vitalité ethnolinguistique." Recherches sociologiques, 2-3: 337-358.

Landry, R., Allard, R. 1987a. "Développement bilingue en milieu minoritaire et en milieu majoritaire." Dans L. Péronnet (Ed.), L'école contribue-t-elle à maintenir la vitalité d' une langue minoritaire? Moncton: Centre de recherche en linguistique appliquée.

Landry, R., Allard, R. 1987b. "Étude du développement bilingue en milieu minoritaire et en milieu majoritaire." Dans. L. Péronnet (Ed.), L'école contribue-t-elle à maintenir la vitalité d' une langue minoritaire? Moncton: Centre de recherche en linguistique appliquée.

Le Blanc, J. 1988. "Un mythe à détruire." Langue et Société. 22: 24-25.

McMahon, F. "Identité franco-albertaine." Rapport de recherche. Edmonton, Alberta. (sous presse).

Morrison, F., Bonyun, R. 1985. Surveys of Bilingual Program Graduates. Eleventh annual report (Part III). Ottawa: Centre de recherches, Le Conseil scolaire d'Ottawa.

Mougeon, R., Heller, M., Beniak, E., Canale, M. 1984. "Acquisition et enseignement du français en situation minoritaire: le cas des Franco-ontariens." La revue canadienne des langues vivantes. 41(2): 315-335.

Richmond, J. 1984. "Superétudiants ou super-problème?" La Revue de l' Université Laurentienne. 17 (1): 113-119.

Société des parents pour les écoles françaises d'Edmonton. 1986-88. Archives, Edmonton, Alberta.

Swain, M., Lapkin, S. 1982. Evaluating Bilingual Education: A Canadian Case Study. Clevedon, Avon: Multilingual Matters, Ltd.

Tardif, C. 1985. "La préparation du matériel didactique et le développement professionnel continu en milieu universitaire." Actes du Colloque national sur l'enseignement postsecondaire en langue française à l' extérieur du Québec. Fédération des francophones hors Québec, Ottawa, 99-103.

Zdunich, L. 1988. A Study of the Number of Students for a Francophone High School. Etude commanditée par la Société des parents pour les écoles françaises d'Edmonton. 